\title{
Editorial
}

\section{What's Lurking Under the Bed? Persistence and Predominance of Particular Clostridium difficile Strains in a Hospital and the Potential Role of Environmental Contamination}

\author{
Lynne V. McFarland, PhD
}

In all cases of infectious disease, determining the method of transmission of the pathogenic agent is paramount for understanding the disease and suggesting appropriate infection control measures. However, the routes of transmission often are difficult to elucidate and document definitively. When I began research on Clostridium difficile in 1985, the method of transmission was not completely understood and the risk factors had just begun to be defined. My first study was about the transmission of and risk factors for nosocomial $C$. difficile on one ward at Harborview Medical Center in Seattle, Washington, during a period of 11 months. ${ }^{1}$ It was a unique study in that, as an unpaid graduate student with no life, I could spend 20 hours a day on the ward tracking patients, doing daily environmental swabs, obtaining specimens for culture from patients and hospital personnel, observing behavior, and running down to the laboratory to prepare the cultures. Because of the daily cultures and persistent patient tracking, solid evidence was collected documenting that $C$. difficile was passed from roommate to roommate, via hospital personnel by hand carriage, and through contaminated environmental vehicles. Serotyping the $C$. difficile strains further unraveled the complexity of the multiple routes of transmission. One $C$. difficile strain, isolated from an index patient admitted with a positive $C$. difficile culture, was found to have contaminated a portable commode chair. This commode chair was responsible for secondary spread to eight other patients on the ward within the span of 1 week. Since that study, numerous other nosocomial outbreaks have been documented and the role of environmental contamination has been verified. ${ }^{2-4}$ Significant strides have been made in this area, but $C$. difficile disease remains a significant problem for hospitalized adults, ${ }^{5,6}$ for pediatric patients, ${ }^{7}$ and, less commonly, in community-acquired cases. 8,9

This issue of Infection Control and Hospital Epidemiology contains two articles on the nosocomial presence of $C$. difficile. ${ }^{10,11}$ Pulvirenti et al. present a study of patients admitted to one of two hospitals in Illinois and describe the prevalence of $C$. difficile strains (as defined by DNA restriction patterns) from patients infected with human immunodeficiency virus (HIV) and environmental sites. ${ }^{10}$ What is interesting about these two hospitals is that although they are both located in Chicago and they both studied only HIV-infected patients using identical culturing methods for $C$. difficile, they had different rates of patients infected with $C$. difficile ( $27 \%$ vs $4 \%$ ) and environmental sites positive for $C$. difficile ( $14.7 \%$ vs $2.9 \%)$. The authors suggest that the higher prevalence at Cook County Hospital may have been due to a greater prevalence of patients with risk factors for $C$. difficile or an increase in numbers as a result of a nosocomial outbreak. The authors tend to downplay the role of environmental contamination in the dissemination of $C$. difficile. A fault in their reasoning is that in their study, environmental cultures were taken only once a month, and during the time of the outbreak, there was a 2-month window when environmental cultures were not taken (February 22 to April 23, 1996). Because the index case became positive on March 13, 1996, environmental sites could have harbored the outbreak strain CD1A for 3 weeks before the index patient was detected. It is impossible to determine the role of environmental contamination in the secondary spread to other cases, as no environmental cultures were taken for approximately 6 weeks 
after the index case was detected. The message here is that without more frequent sampling of the environment, it is impossible to determine which came first, the infected patient or the contaminated environment. It is always a full moon when you look up at the sky only once a month.

If their data are viewed from a different perspective, the frequency of $C$. difficile isolation actually points to the important role of environmental contamination. Cook County Hospital, which had the higher rate of $C$. difficile-positive patients (27\%) and environmental sites (14.7\%), also had a greater degree of shared rooms and communal areas (bathrooms, sinks, and lounge areas) and higher numbers of hospital transfers. All of these lend themselves to easier transmission via contaminated environmental sites. Patients and hospital personnel may act as a movable feast for the transmission of nosocomial $C$. difficile. ${ }^{1,4,5}$ The authors point out that nosocomial outbreaks of C. difficile start with an infected index patient, but downplay the important role that environmental contamination has in the secondary spread of $C$. difficile in hospitals. This ignores the role that environmental disinfection practices have in controlling nosocomial outbreaks of $C$. difficile. ${ }^{12,13}$

In the other article, Mekonen et al. found that the same restriction endonuclease analysis (REA) group $\mathrm{K}$ was the most predominate $C$. difficile strain in two Chicago hospitals, although there were no shared patients or personnel. ${ }^{11}$ The authors speculate that there may be regional strains of $C$. difficile that tend to have mild genetic drift once they become established in one hospital. Because one of the hospitals (Cook County Hospital) was the same for both studies, it is reasonable to try to compare the results of these two studies. Is $C$. difficile type CD1A from one study $^{10}$ the same $C$. difficile REA group $\mathrm{K}$ in the other study? ${ }^{11}$ However, the $C$. difficile strains were determined using different typing methods, so the results are not comparable. This raises another issue about the value of having numerous strain typing methods for each study of $C$. difficile disease. What is needed is a Rosetta stone for the dif- ferent strain typing procedures so that we can compare the results of different studies on $C$. difficile.

Although research on nosocomial $C$. difficile has come far since the late $1970 \mathrm{~s}, C$. difficile lives up to its name by continuing to be one of the most difficult clostridial infections in our hospitals.

\section{REFERENCES}

1. McFarland LV, Mulligan ME, Kwok RYY, Stamm WE. Nosocomial acquisition of Clostridium difficile infection. $N$ Engl J Med 1989;320:204210.

2. Cohen SH, Tang YJ, Rahmani D, Silva J. Persistence of an endemic (toxigenic) isolate of Clostridium difficile in the environment of a general medicine ward. Clin Infect Dis 2000;30:952-954.

3. Kroker PB, Bower M, Azadian B. Clostridium difficile infection, hospital geography and time-space clustering. Q I Med 2001;94:223-225.

4. Samore MH, Venkataraman L, DeGirolami PC, Arbeit RD, Karchmer AW. Clinical and molecular epidemiology of sporadic and clustered cases of nosocomial Clostridium difficile diarrhea. Am J Med 1996; 100:32-40.

5. Lai KK, Melvin S, Menard MJ, Kotilainen HR, Baker S. Clostridium difficile-associated diarrhea: epidemiology, risk factors and infection control. Infect Control Hosp Epidemiol 1997;18:628-632.

6. Johnson S, Samore MH, Karrow KA, et al. Epidemics of diarrhea caused by a clindamycin-resistant strain of Clostridium difficile in four hospitals. N Engl J Med 1999;341:1645-1651.

7. McFarland LV, Brandmarker SA, Guandalini S. Pediatric Clostridium difficile: a phantom menace or clinical reality? J Pediatr Gastroenterol Nutr 2000:31:220-231.

8. Levy DG, Stergachis A, McFarland LV, et al. Antibiotics and Clostridium difficile diarrhea in the ambulatory care setting. Clin Ther 2000;22:91102.

9. Hirschhorn LR, Trnka Y, Onderdonk A, Lee MT, Platt R. Epidemiology of community-acquired Clostridium difficile-associated diarrhea. J Infect Dis 1994;169:127-133.

10. Pulvirenti JJ, Gerding DN, Nathan C, et al. Difference in the incidence of Clostridium difficile among patients infected with human immunodeficiency virus admitted to a public and a private hospital. Infect Control Hosp Epidemiol 2002;23:641-647.

11. Mekonen ET, Gerding DN, Sambol SP, et al. Predominance of a single restriction endonuclease analysis group with intrahospital subgroup diversity among Clostridium difficile isolates at two Chicago hospitals. Infect Control Hosp Epidemiol 2002;23:648-652.

12. Wilcox MH, Fawley WN. Hospital disinfectants and spore formation by Clostridium difficile. Lancet 2000;356:1324.

13. Mayfield JL, Leet T, Miller J, Mundy LM. Environmental control to reduce transmission of Clostridium difficile. Clin Infect Dis 2000;31:9951000 . 